Au cours de la purification de l'inhibine, une fraction, séparée par chromatographie, s'est révélée avoir un effet biologique inverse de celui de cette hormone: une stimulation spécifique de la libération de la FSH par les cellules antehypophysaires, sans influence sur la sécrétion de la LH ni des autres hormones hypophysaires [I, 2].

Deux équipes californiennes, celles de Roger Guillemin [I] et de Wylie Vale [2], ont réussi à purifier totalement des substances responsables de cet effet activateur de la sécrétion de FSH. Il s'agit dans les deux cas d'une protéine dimérique, les deux sous-unités étant liées par un pont disulfure.

L'analyse partielle de la séquence en acides aminés devait indiquer que l'hormone isolée par l'équipe de Roger Guillemin, dénommée " activine ", était composée des sousunités $\beta_{A}$ et $\beta_{B}$ de l'inhibine. L'hormone isolée par le laboratoire de Wylie Vale, désignée par les initiales FRP (FSH Releasing Protein) était, elle, un homodimère composé de deux sous-unités $\beta_{A}$ de l'inhibine (figure I). La concentration de ces deux substances provoquant une sécrétion demi-maximale de FSH par les cellules antéhypophysaires était de I 50 pmoles/litre pour l'activine et 25 pmoles/litre pour le FRP. Alors même que la signification physiologique de la production par l'ovaire d'inhibine, d'activine et de FRP reste obscure et que l'on ne sait pas si d'autres tissus, l'hypophyse et l'hypothalamus notamment, sécrètent ces substances, le modèle de contrôle hormonal par une petite quantité de molécules variablement associées que suggèrent ces résultats, est potentiellement important (figure I).

A.K.

I. Ling $N$, Ying SY, Veno $\mathrm{N}$ et al. Pituitary FSH is released by a heterodimer of the $\beta$ subunits from the two forms of inhibin. Nature 1986; $321: 779-82$

2. Vale W, Rivier J, Vaughan J et al. Purification and charactcrization of an FSH relcasing protein from porcine ovarian follicular fluid. Nature 1986 ; $321: 776-9$.

$\mathrm{m} / \mathrm{s} n^{\circ} 8$, vol 2, octobre 86

\title{
Des inhibiteurs
}

\section{de la croissance cellulaire}

\author{
"Transforming Growth Factor $\beta$ " \\ (le mal nommé) \\ et hormone antimüllerienne
}

Antioncogènes [I], facteur de vieillissement (médecine/science, $\mathrm{n}^{\circ} 7$, vol. 2, p. 404), inhibiteurs de la croissance, " transforming growth factor $\beta "(T G F-\beta)[2]$, hormone antimüllerienne [3], du général à l'individuel et sous plusieurs dénominations, les agents s'opposant à la croissance et à la prolifération cellulaire font une entrée rapide sur la scène de la biologie moderne. Le concept de "Chalones" prédisant l'existence de substances s'opposant à la croissance cellulaire est ancien mais les premières mises en évidence de tels agents antiprolifératifs sont une retombée des techniques de la génétique somatique : un hybride formé par la fusion d'une cellule normale et d'une cellule cancéreuse perd souvent son potentiel invasif lorsqu'il est transféré dans la souris immunodéprimée [4]. La récupération du pouvoir invasif de ces hybrides est correlée avec la perte de certains chromosomes, notamment du chromosome II dans le cas particulier d'une fusion entre des fibroblastes et des cellules dérivées d'un cancer du col utérin (lignée Hela). Ce même chromosome II, Claudine Funien l'a récemment rappelé dans ces colonnes [I], est impliqué dans un syndrome malformatif héréditaire (syndrome de Beckmith-Wiedeman) se compliquant très fréquemment de trois types de tumeur, le néphroblastome (tumeur rénale de Wilms), l'hépatoblastome (tumeur hépatique), et le rhabdomyosarcome (tumeur du muscle strié). Dans ces tumeurs, on retrouve une lésion des deux chromosomes II, alors qu'un seul est atteint dans les autres cellules de malade. Ces données suggèrent par conséquent que les malades atteints d'un syndrome de Beckmith-Wiedeman seraient hétérozygotes pour une mutation à un locus dont le défaut homozygote, qui pourrait être acquis par une mutation somatique supplémentaire, entraînerait le blocage de différenciation et la prolifération de certains tissus. Rien ne dit que ce locus, qui pourrait correspondre à un gène codant pour un "antioncogène ", soit également celui responsable de la récupération $d u$ pouvoir prolifératif d'hybrides entre des cellules normales et des cellules cancéreuses ayant perdu le chromosome II. La réintroduction d'un chromosome II normal dans ces hybrides aussi bien que dans des cellules de tumeur de Wilms serait cependant, selon des résultats encore préliminaires [4], capable de supprimer leur tumorigénicité, indiquant qu'il porte bien un ou plusieurs types d'informations anti-prolifératives. D'autres résultats très récents indiquent que la transformation maligne d'une cellule par la combinaison d'un oncogène "immortalisant" (tel c-myc) et d'un oncogène "transformant" (tel c-ras) requiert, en plus, la perte de tout ou partie d'un chromosome qui pourrait coder pour une information suppressive.

Quelles pourraient-être les substances synthétisées sous la direction de tels gènes suppresseurs de la prolifération, ou anti-oncogènes? L'une d'entre elles, bien mal nommée, est le TGF- $\beta$ (Transforming Growth Factor $\beta$ ), d'abord identifié par sa capacité à stimuler la prolifération des fibroblastes. Cet effet stimulateur est en fait dû̀ $a$ une induction secondaire de la synthèse d'un facteur de croissance, le PDGF (Platelet Derived Growth

. .

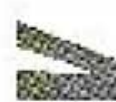


Factor), et le TGF- $\beta$ est le plus souvent un suppresseur de la prolifération. Il est synthétisé sous la forme d'une chaîne protéique inactive de 391 acides aminés, secondairement clivée en sous-unités actives de II 2 résidus, le produit final étant formé de deux de ces sous-unités. Certains exemples sont connus où le potentiel prolifératif de lignées cancéreuses est associé soit à l'impossibilité de transformer le précurseur de 39 I acides aminés en la forme active, soit à l'absence des récepteurs membranaires $d u T G F-\beta$, indispensables à son action. Les interférons constituent également une classe de substances antiprolifératives s'opposant notamment à l'expression de certains oncogènes. L'interféron $\beta$ semble ainsi jouer un rôle déterminant dans le contrôle de l'expression de l'oncogène c-myc au cours de la différenciation terminale des cellules hématopoḯtiques [5]. L'hormone anti-müllérienne $(A M H$, AntiMüllerian Hormone) peut, elle aussi, être considérée comme protéine antiproliférative, responsable de la régression des canaux de Müller chez les embryons mâles chez lesquels elle est synthétisée par les cellules testiculaires de Sertoli [6]. Elle pourrait aussi inhiber la prolifération de lignées cancéreuses dérivées d'endomètres et d'ovaires [6]. Deux équipes, l'une américaine et l'autre française [3, 8], viennent de "cloner" l'ADN complémentaire du messager de cette hormone, et donc d'élucider la structure complète de la protéine. Il apparaît que la partie carboxy-terminale de l'AMH est significativement homologue au $T G F-\beta$, suggérant que le gène de l'AMH a pu être formé dans l'évolution par ce mécanisme de redistribution des exons (exon shuffling) dont nous avons parlé ici même (médecine/science, $\mathrm{n}^{\circ} \mathrm{I}$, vol. 2 , p. 5I).Le domaine de l'AMH ressemblant au $T G F-\beta$ et le TGF- $\beta$ lui-même, dériveraient d'un gène ancestral qui pourrait avoir déjà eu un rôle d'inhibition de la prolifération. L'origine de l'AMH [g] et du TGF- $\beta$ pourrait être une duplication ancienne du gène ancestral suivie de l'acquisition par chacun des deux nouveaux gènes de nouvelles "particules d'informations "... sous la forme d'exons, conférant aux protéines correspondantes des spécificités d'action différentes. Il faut noter que les gènes de $I A M H$ et du TGF- $\beta$ sont tous deux situés sur le chromosome 19, mais en des régions différentes [9]. Les oncogènes sont "nés" en 1976 et chacun voit ce que fut leur "développement ". Voici maintenant qu'apparaît le concept d'antioncogène... "le Yin et le Yang " selon l'expression du Dr Marx [4]. Souhaitons d'autant plus vivement longue vie aux "nouveaux-nés " que si les oncogènes nous ont permis de comprendre le cancer... les antioncogènes nous font percevoir comment le combattre.

A.K.

I. Junien C. Les antioncogènes. médecine/sciences, 1986; $2: 238-45$

2. Roberts AB, Anzano MA, Wakefield LM, Roche NS, Stern DF, Sporn MB. Type B transforming growth factor: a bifunctional regulatory of cellular growth. Proc Natl Acad Sci USA 1985; 82 : $119-23$.

3. Cate RL, Mattaliano RJ, Hession C et al. Isolation of the bovine and human genes for müllerian inhibiting substance and expression of the human gene in animal cells. Cell 1986; $45: 685$ , ty

4. Marx JL. The "Yin and Yang" of cell growth control. Science 1986; 232 : 1093-5.

5. Resnitzky D, Yarden A, Zipori D, Kimchi A Autocrine $\beta$-related interferon controls $c$-myc suppression and growth arrest during hematopoietic cell differentiation. Cell 1986; 46 31-40.

6. Josso $\mathrm{N}$. In vitro synthesis of müllerian inhibiting substance hormone by seminiferous tubules isolated from the calf fetal testis. Endocrinology 1973; $93: 829-34$.

7. Füller AF, Krane IM, Budzik GP, Donahoe PK. Müllerian inhibiting substance reduction of colony growth of human gynecologic cancers in a stem cell assay. Gynecol Oncol 1985; 22 : 135-48.

8. Picard JY, Benarous R, Gucrrier D, Josso N, Kahn A. Cloning and expression of cDNA for anti-müllerian hormonc. Proc Natl Acad Sci USA 1986; 83 : 5464-8.

9. Cohen-Haguenauer O, Picard JY, Serero S, et al. Mapping of the gene for anti-müllerian hormone to human chromosome 19. Cytogenet Cell Genet 1986; (sous presse).

\section{COURRIER}

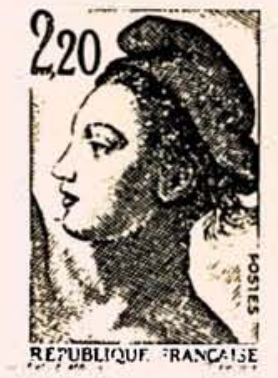

Nous avons reçu du Docteur Marc Piechaczyk (Laboratoire du docteur Jeanteur de Montpellier) les précisions suivantes à la suites de la nouvelle intitulée "régulation post-transcriptionnelle des oncogènes" $\left(m / s \mathrm{n}^{\circ} 2\right.$, vol. 2, p. 286):

"Bien que le point ne soit pas d'une importance capitale, j'aimerais lever toute ambiguïté sur le rôle du premier exon non codant de l'oncogène $\mathrm{c}$-myc. Il est vrai que les ARNm dépourvus de l'exon I sont significativement plus stables que leurs homologues normaux, mais il apparait, comme nous en évoquions la possibilité dans Piechaczyk et al. Cell 1985; 42 : 589 , que l'incrément de stabilité résulte en fait de l'addition de séquences étrangères. De plus, nous avons clairement montré que le I ${ }^{\text {er }}$ exon par lui-même est incapable de conférer une instabilité quelconque à des séquences hétérologues (Eick et al. EMBO 7 1985; Piechaczyk et al. CTMI I986 (sous presse). Au contraire, différentes observations indiquent que: (a) les régions responsables de l'instabilité seraient situées dans la partie $z^{\prime}$ terminale de l'ARNm comme pour l'oncogène c-fos, ainsi que l'ont suggéré les travaux de Treisman et les nôtres (soumis pour publication); (b) la structure globale de l'ARNm c-myc est un paramètre important pour sa dégradation rapide (article en préparation).» 